\title{
Theory of Smith-Purcell radiation from a 2D array of small noninteracting particles
}

\author{
D. I. Garaev $\odot,{ }^{1}$ D. Yu. Sergeeva $\odot,{ }^{1,2}$ and A. A. Tishchenko $\circledast^{1,2,3, *}$ \\ ${ }^{1}$ National Research Nuclear University “MEPhI,” Moscow, Russia \\ ${ }^{2}$ Laboratory of Radiation Physics, Belgorod National Research University, Belgorod, Russia \\ ${ }^{3}$ National Research Center “Kurchatov Institute," Moscow, Russia
}

(Received 12 October 2020; revised 10 January 2021; accepted 11 January 2021; published 1 February 2021)

\begin{abstract}
We construct the first-principles theory for the scattering of the Coulomb field of a fast electron traveling along an array of nano- or microparticles. The electron's trajectory is arbitrarily oriented in the plane parallel to the surface. We show that Smith-Purcell radiation accompanying this process results in very rich diffraction patterns, which differ dramatically from the ones for conventional diffraction gratings; a numerical analysis was made for THz frequencies. The generalized Smith-Purcell dispersion relation has been derived; it describes the link between frequency, two periods of the structure, the electron's velocity, and the angle of radiation with maximal intensity. The unique spatial distribution of the generated light can form the basis for the generation of structured-light electron-driven photon sources.
\end{abstract}

DOI: 10.1103/PhysRevB.103.075403

\section{INTRODUCTION}

Smith-Purcell radiation (SPR) is emitted when a charge moves near a periodically inhomogeneous target. Purcell and Smith first measured it in 1953 [1] and since then SPR has been studied for different gratings and in various frequency ranges (see the references in monographs [2,3]). The main feature of SPR is its dispersion relation:

$$
d\left(\beta^{-1}-\cos \psi\right)=s \lambda, \quad s=1,2, \ldots,
$$

with $d$ being the period of the grating, $\lambda$ being the wavelength of radiation; $\beta=v / c$, where $c$ is the speed of light in vacuum and $v$ is the velocity of the electron; $\psi$ is the angle of observation relative to the electron trajectory; see Fig. 1. The correspondence of each wavelength to each observation angle in Eq. (1) means monochromaticity of SPR and possible large observation angles.

Compared with traditional optics, the way of exciting radiation using SPR is attractive due to (i) the opportunity to excite electromagnetic waves in a wide spectral range (as the electrons bring the Coulomb field that contains the frequencies of wide range); (ii) absence of a feeding system for an emitting antenna, which helps to avoid extra complexity of the whole system; and (iii) possibility to tune the characteristics of radiation changing the energy of the electron beam, etc.

Besides its customary applications in powerful orotronlike sources of radiation and in nondestructive diagnostics of relativistic electron beams, during the last decade SPR has been intensively investigated for more exotic structures, such as plasmonic crystals [4-6], graphene nanoscale gratings [7-9], and metasurfaces [10-13].

Mostly, the theory involved in the analysis is restricted to the use of dispersion relations (the Smith-Purcell one,

\footnotetext{
*alexey.tishchenko@cern.ch
}

the plasmons one, etc.), while the analytical expressions describing dependence of the radiation field or intensity on the parameters of the beam and of the target are rare (references up to 2010 can be found in $[2,4]$; the cycle of research by Garcia de Abajo with coauthors is of interest in this regard [5,14]). Also, Glass in [15] developed a theory of SPR from bigratings; his theory is impressively general, though it is seminumerical and valid only for metal gratings (and, in the IR range, for semiconductors) and for bigratings comprised of periodically arranged bumps consisting of the same material as the substrate.

The theory of SPR was developed in $[16,17]$ for flat arrays of nano- and microparticles. The theory in Ref. [16] is valid for flat rectangular two-dimensional (2D) arrays of subwavelength particles when the electron's trajectory coincides with one of two directions in which the target is periodic. In practice, however, there is a distortion of the electron's trajectory caused either by electron beam divergence or by not high enough accuracy in the beam positioning. Therefore, to have the theory valid for a wide range of parameters is principally important, and the question arises: Can such a theory be obtained from the one developed before by a relatively simple replacement or substitution? Below we will show that for surfaces with two periods the correct answer is "no."

As for radiation from a conventional diffraction grating, the oblique incidence of an electron leads to increasing the period by the factor $1 / \cos \chi$; see Fig. 2. Consequently, the dispersion relation in Eq. (1) transforms [18] into

$$
\frac{d}{\cos \chi}\left(\beta^{-1}-\cos \psi\right)=s \lambda, \quad s=1,2, \ldots
$$

For a conventional grating this deviation leads to a strong spatial redistribution of SPR $[18,19]$ called the "conical effect." This is angular redistribution of SPR maxima from a plane to a conical surface with the axis along strips and the generator close to the electron trajectory (compare Figs. 1 


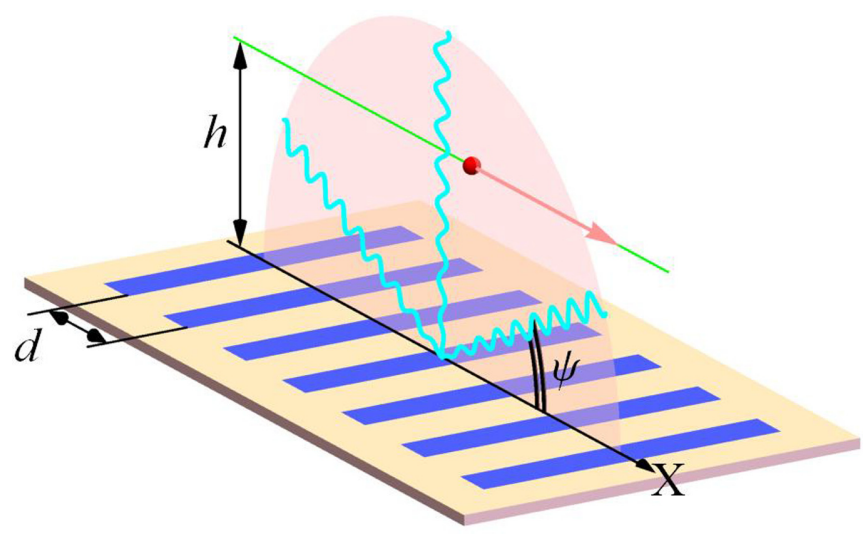

FIG. 1. Generation of SPR by an electron moving near a conventional diffraction grating.

and 2). This effect was discussed in [19], analytically described in [18], and experimentally proved in [20].

The situation changes for two-periodic targets consisting of small particles (Fig. 3): Now the oblique incidence results not in mere lengthening of the period, but in the modification of the arrangement of inhomogeneity along the electron trajectory; see Fig. 4. This should lead to complication of the diffraction pattern and therefore to an essentially different dispersion relation.

This arrangement can be similar to the quasicrystals. Indeed, the radiation is emitted mainly from the area "illuminated" by the Coulomb field of the electron under its trajectory (gray area in Fig. 4). The projection of particles emitting radiation on the electron trajectory can form a quasicrystal, so the total radiation can be considered as the radiation from a quasicrystal [21,22]. Among other aperiodic chains SPR from a one-dimensional quasicrystal was calculated and analyzed by Saavedra et al. in [14]. They show that the angular distribution of radiation from such a target is well ordered but is more complex than that from a strongly periodic target.

For two-dimensional targets, the emerging aperiodicity means that the existing theory for normal incidence of the electron cannot be directly extended to the case of oblique incidence, and therefore a separate calculation is required.

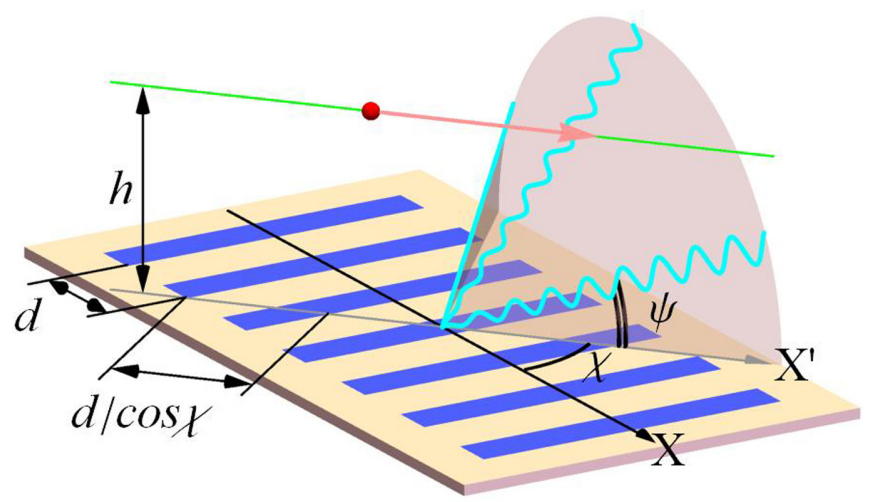

FIG. 2. Generation of SPR by an electron moving near a conventional diffraction grating at arbitrary angle $\chi$ (oblique incidence).

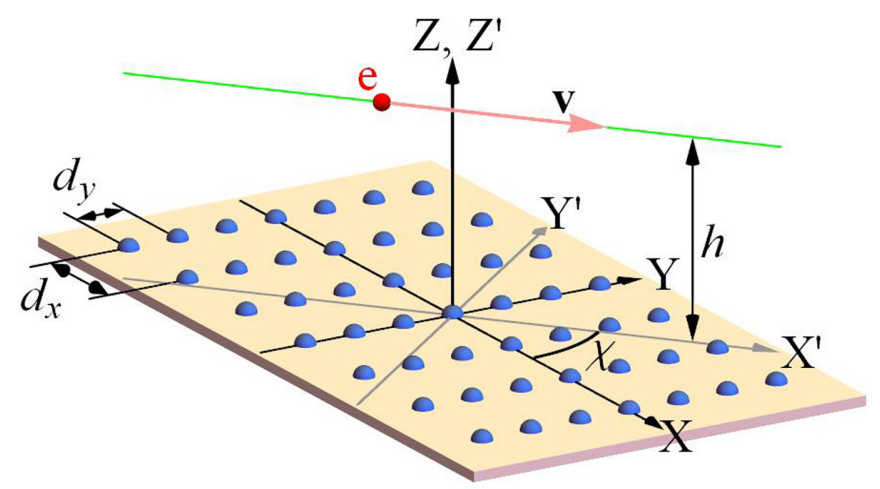

FIG. 3. Generation of SPR by an electron moving near 2D periodic array of subwavelength particles at arbitrary angle (oblique incidence).

Below, we derive the fully analytical theory of radiation for electrons passing over a two-periodic array of subwavelength particles for arbitrary angle between the electron trajectory and directions of periodicity of the 2D array. As we will see, introducing the additional parameter-arbitrary angle $\chi$-leads to a dramatic change in the diffraction patterns compared with those for a conventional diffraction grating, and the generalized dispersion relation must be used to describe it.

\section{RADIATION FIELD AND INTENSITY}

In this section, we state a theory of radiation generated by an electron passing over a $2 \mathrm{D}$ periodic array. The array is a finite number of identical small particles having characteristic size $r_{0}$ and arranged as a 2D rectangular lattice. The particles can be of any form, including holes - what is important is that their size is less than the wavelength. The coordinate system is chosen so that $d_{x}$ and $d_{y}$ are the periods of the grating along the $O X$ and $O Y$ axes, correspondingly. Counting the objects by the index $m=\left\{m_{x}, m_{y}\right\}$, which is the set of two integers $m_{x}$ and $m_{y}$, one can define the coordinates of the particles as

$$
\begin{aligned}
\mathbf{R}_{m} & =d_{x} m_{x} \mathbf{e}_{x}+d_{y} m_{y} \mathbf{e}_{y}, \\
m_{x} & \in\left[-M_{x}, M_{x}\right], \quad m_{y} \in\left[-M_{y}, M_{y}\right] .
\end{aligned}
$$

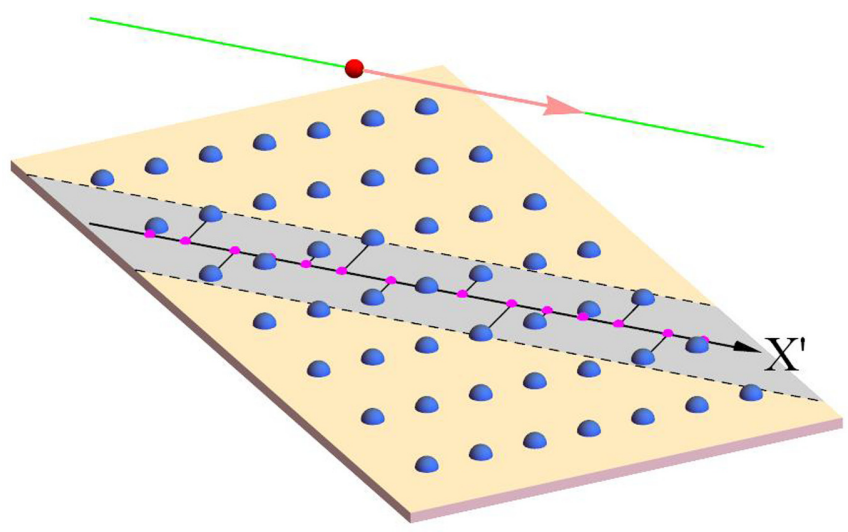

FIG. 4. The projection of particles that emit radiation on the electron trajectory. The gray area is one illuminated by the Coulomb field of the electron. 
A free electron travels parallel to the target plane with the velocity $\mathbf{v}$ and impact parameter $h$ being the shortest distance between the electron and the plane. The projection of the velocity on the plane $O X Y$ makes an angle $\chi$ with the $O X$ axis, i.e., $\mathbf{v}=v(\cos \chi, \sin \chi, 0)$; see Fig. 3 .

To calculate the field of radiation we solve the Maxwell's equations and then express the field components through the current density of the electron. The Fourier image for the solution of the Maxwell's equations is (see, e.g., [2])

$$
\mathbf{E}(\mathbf{r}, \omega)=-\frac{4 \pi i}{\omega} \int d \mathbf{q} e^{i \mathbf{q r}} \frac{\mathbf{q}\left(\mathbf{q}, \mathbf{j}^{\mathrm{tot}}(\mathbf{q}, \omega)\right)-k^{2} \mathbf{j}^{\mathrm{tot}}(\mathbf{q}, \omega)}{q^{2}-k^{2}},
$$

where the frequency $\omega$, the velocity of light in vacuum $c$, and the wave number $k$ are related by $k=\omega / c$.

The total current density $\mathbf{j}^{\text {tot }}$ consists of the current density of the free electron $\mathbf{j}^{0}(\mathbf{r}, t)=e \mathbf{v} \delta(\mathbf{r}-\mathbf{v} t)$ and the current density $\mathbf{j}$ induced in the target: $\mathbf{j}^{\text {tot }}=\mathbf{j}^{0}+\mathbf{j}$.

For the wavelengths $\lambda \gg r_{0}$ in the dipole approximation the current density $\mathbf{j}$ reads

$$
\mathbf{j}(\mathbf{r}, \omega)=-i \omega \sum_{m} \mathbf{d}\left(\mathbf{R}_{m}, \omega\right) \delta\left(\mathbf{r}-\mathbf{R}_{m}\right),
$$

where $\delta$ is the Dirac delta function; $\mathbf{d}$ is the dipole moment, which for $\lambda \gg r_{0}$ can be expressed through the polarizability $\alpha(\omega)$ :

$$
\mathbf{d}\left(\mathbf{R}_{m}, \omega\right)=\alpha(\omega) \mathbf{E}^{0}\left(\mathbf{R}_{m}, \omega\right) .
$$

Here the sum over all $m$ means the double sum over $m_{x}$ and $m_{y}: \sum_{m}(\cdots)=\sum_{m_{x}} \sum_{m_{y}}(\cdots)$. When writing down Eq. (6) we replaced the field acting to the particle by the Coulomb field of electron $\mathbf{E}^{0}(\mathbf{r}, \omega)$. This approximation means that the fields from the neighboring particles are neglected. Then the Fourier transform of the current density is

$$
\mathbf{j}(\mathbf{q}, \omega)=-\frac{i \omega}{(2 \pi)^{3}} \alpha(\omega) \sum_{m} \mathbf{E}^{0}\left(\mathbf{R}_{m}, \omega\right) e^{-i \mathbf{q} \mathbf{R}_{m}} .
$$

Therefore, Eq. (4), when written for components, reads

$$
\begin{aligned}
E_{i}(\mathbf{r}, \omega)= & E_{i}^{0}(\mathbf{r}, \omega)-\frac{\alpha(\omega)}{2 \pi^{2}} \int d^{3} q \frac{q_{i} q_{j}-k^{2} \delta_{i j}}{q^{2}-k^{2}} \\
& \times \sum_{m} E_{j}^{0}\left(\mathbf{R}_{m}, \omega\right) e^{i \mathbf{q}\left(\mathbf{r}-\mathbf{R}_{m}\right)}
\end{aligned}
$$

where $\delta_{i j}$ is the Kronecker delta.

Knowing the formula for the Coulomb field of the electron in a system $K^{\prime}$ (see Fig. 2), in which it moves along the $O X^{\prime}$ axis, $\mathbf{E}^{\prime 0}\left(\mathbf{r}^{\prime}, \omega\right)$, we can find $\mathbf{E}^{0}(\mathbf{r}, \omega)$ rotating vectors by the angle $\chi$ about the $O Z$ axis: $\mathbf{E}^{0}(\mathbf{r}, \omega)=A \mathbf{E}^{\prime 0}\left(A^{-1} \mathbf{r}, \omega\right)$, where $A^{-1}$ is the inverse matrix $A$ :

$$
A=\left(\begin{array}{ccc}
\cos \chi & -\sin \chi & 0 \\
\sin \chi & \cos \chi & 0 \\
0 & 0 & 1
\end{array}\right)
$$

Applying this transformation we find

$$
\mathbf{E}^{0}(\mathbf{r}, \omega)=-\frac{e \omega}{\pi v^{2} \gamma} e^{\frac{i \omega}{v^{2}} \mathbf{v R}}\left\{\frac{i}{\gamma} K_{0}\left(L \frac{\omega}{v \gamma}\right) \frac{\mathbf{v}}{v}+\frac{\mathbf{L}}{L} K_{1}\left(L \frac{\omega}{v \gamma}\right)\right\},
$$

where $\mathbf{L}=\left[\mathbf{v},\left[\mathbf{v}, \mathbf{r}-h \mathbf{e}_{z}\right]\right] / v^{2}, e$ is the charge of the electron, $\gamma$ is the Lorentz factor of the electron, and $K_{0}$ and $K_{1}$ are modified Bessel functions of the second kind of the zero and the first order, correspondingly.

The field of radiation $\mathbf{E}^{\mathrm{rad}}(\mathbf{r}, \omega)$ is the total field $\mathbf{E}(\mathbf{r}, \omega)$ in the far zone $(k r \gg 1)$ minus the Coulomb field $\mathbf{E}^{0}(\mathbf{r}, \omega)$. So, after integrating in Eq. (8) we have

$$
\begin{aligned}
\left.\mathbf{E}^{\mathrm{rad}}(\mathbf{r}, \omega)\right|_{k r \gg 1}= & \alpha(\omega) \frac{e \omega}{\pi c^{2} \beta^{2} \gamma} \frac{e^{i k r}}{r} \\
& \times \sum_{m} e^{i d_{x} m_{x} \varphi_{x}} e^{i d_{y} m_{y} \varphi_{y}}\left[\mathbf{k},\left[\mathbf{k}, \mathbf{P}_{m}\right]\right],
\end{aligned}
$$

where $k_{x}, k_{y}$ are the corresponding components of the wave vector $\mathbf{k}=k \mathbf{n}$, and $\mathbf{n}$ is the unit wave vector,

$$
\begin{aligned}
\varphi_{x} & =\frac{\omega v_{x}}{v^{2}}-k_{x}, \quad \varphi_{y}=\frac{\omega v_{y}}{v^{2}}-k_{y}, \\
\mathbf{P}_{m} & =\frac{i}{\gamma} \frac{\mathbf{v}}{v} K_{0}\left(L_{m} \frac{\omega}{v \gamma}\right)+\frac{\mathbf{L}_{m}}{L_{m}} K_{1}\left(L_{m} \frac{\omega}{v \gamma}\right), \\
\mathbf{L}_{m} & =\left[\mathbf{v},\left[\mathbf{v}, \mathbf{R}_{m}-h \mathbf{e}_{z}\right]\right] / v^{2} .
\end{aligned}
$$

Then, the emitted energy of radiation per unit photon energy $d \hbar \omega$ and per solid angle $d \Omega$ reads

$$
\frac{d W(\mathbf{n}, \omega)}{d \hbar \omega d \Omega}=\frac{c r^{2}}{\hbar}\left|\mathbf{E}^{\mathrm{rad}}(\mathbf{r}, \omega)\right|^{2} .
$$

Substituting Eq. (11) into Eq. (15) we obtain

$$
\begin{aligned}
\frac{d W(\mathbf{n}, \omega)}{d \hbar \omega d \Omega}= & \frac{e^{2}}{\hbar c} \frac{1}{\pi^{2}} \frac{\omega^{4}|\alpha(\omega)|^{2}}{c^{4} \beta^{4} \gamma^{4}} \mid \sum_{m} e^{i d_{x} m_{x} \varphi_{x}} e^{i d_{y} m_{y} \varphi_{y}} \\
& \times\left.\left[\mathbf{k}, \frac{\mathbf{v}}{v} K_{0}\left(L_{m} \frac{\omega}{v \gamma}\right)-i \gamma \frac{\mathbf{L}_{m}}{L_{m}} K_{1}\left(L_{m} \frac{\omega}{v \gamma}\right)\right]\right|^{2} .
\end{aligned}
$$

When the electron moves parallel to the $O X$ axis, i.e., for $\chi=0$, Eq. (16) coincides with that from [16]. In the case of a chainlike target, i.e., for $\chi=0$ and $M_{y}=0$, the obtained result coincides with the result from [23].

Note that all the theory developed here does suppose that the single particles composing the array do not interact. This approximation holds true when the distance between them significantly exceeds their sizes; see [24].

In the theory constructed by Glass [15], the radiated power is expressed through the coefficients that are to be obtained from the infinite set of linear equations (see the discussions before Eq. (20) and around Eqs. (56) and (57) in [15]), contrary to our Eq. (16), which is fully analytical. Besides, we can consider the restricted gratings, while Glass's theory describes the radiation from the gratings infinite in the transversal direction. Instead of that, Glass's theory allows for surface plasmon excitation in contrast with Eq. (16), which describes the radiation in the far zone only.

\section{DISPERSION RELATIONS}

As modified Bessel functions are monotonous, the maxima of the radiation are mainly defined by exponents in the sum in Eq. (16). The next system of two dispersion relations is 
obtained from Eq. (16),

$$
\begin{aligned}
& \lambda s_{x}=d_{x}\left(\frac{\beta_{x}}{\beta^{2}}-n_{x}\right), \\
& \lambda s_{y}=d_{y}\left(\frac{\beta_{y}}{\beta^{2}}-n_{y}\right),
\end{aligned}
$$

with $s_{x}$ and $s_{y}$ being integers, and $\beta_{x, y}=v_{x, y} / c$. The observation angles are defined as follows:

$$
n_{x}=\cos \theta, \quad n_{y}=\sin \theta \sin \phi, \quad n_{z}=\sin \theta \cos \phi .
$$

Unlike the case of conventional diffraction grating, these two dispersion relations cannot be obtained from those for normal motion of the electron by adjusting the period following the rule discussed above, such as $d \rightarrow d / \cos \chi$; see Fig. 2. Taking into account that the components of the unit wave vector $n_{x}$ and $n_{y}$ are less than unity, we find the limits for the integers $s_{x}$ and $s_{y}$ from Eq. (17):

$$
\begin{aligned}
& \frac{d_{x}}{\lambda}\left(\frac{\beta_{x}}{\beta^{2}}-1\right) \leqslant s_{x} \leqslant \frac{d_{x}}{\lambda}\left(\frac{\beta_{x}}{\beta^{2}}+1\right), \\
& \frac{d_{y}}{\lambda}\left(\frac{\beta_{y}}{\beta^{2}}-1\right) \leqslant s_{y} \leqslant \frac{d_{y}}{\lambda}\left(\frac{\beta_{y}}{\beta^{2}}+1\right) .
\end{aligned}
$$

For $\beta_{y}=0$ we have $\beta_{x}=\beta$, and the first equation of the two in Eq. (17) turns into the conventional dispersion relation for SPR from Eq. (1) with only positive integers $s_{x}$. In this case the second one in Eq. (17) goes to the additional dispersion relation caused by the second period, coinciding with the formula (2.15) from [17]:

$$
\lambda s_{y}=-n_{y} d_{y} .
$$

For $s_{y}=0$ the second relation in Eq. (17) reads

$$
n_{y}=\beta_{y} / \beta^{2} \text {. }
$$

Equation (21) coincides with the equation for the conical surface of maximal SPR from conventional gratings (so-called "conical effect") [18]; this cone has its axis directed along the $y$ axis (see Fig. 2). The essence of it is in the spatial distribution of SPR over a conical surface for the case of oblique movement of the electron above the grooves of the grating. We have justified another explanation in [25]: Equation (21) coincides with the condition of Cherenkov radiation from a superluminal source, which is the area of the target excited by the Coulomb field of the moving electron.

For $s_{x}=0$ we obtain a similar equation for the conical surface of the cone with its axis directed along the $x$ axis. For $s_{y}=0$ from Eq. (21) and the first equation of the two in Eq. (17) we find

$$
\lambda s=\frac{d_{x}}{\beta_{x}}\left(1-\beta_{x} n_{x}-\beta_{y} n_{y}\right), \quad s=0,1,2 \ldots,
$$

which coincides with the dispersion relation for SPR for a conventional grating [18]. Actually, Eq. (22) can easily transform into Eq. (2) provided that $\chi$ is the angle between the vector $\mathbf{v}$ and the $x$ axis, and $\psi$ is the angle between the vectors $\mathbf{v}$ and $\mathbf{k}$.

Besides, it was shown in [18] that the dispersion relation for SPR from a conventional grating is similar to that for the plane wave diffracted on the grating, with the only difference

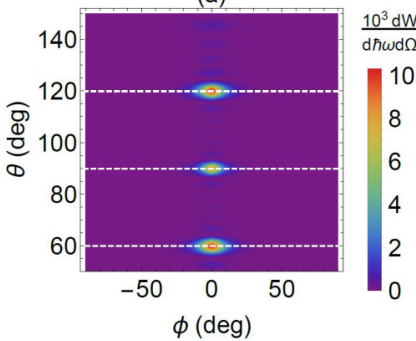

(b)

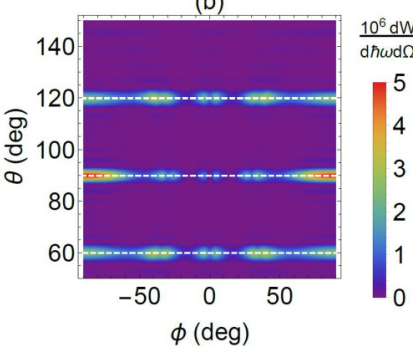

FIG. 5. Angular dependences of spectral-angular distribution of SPR from a conventional grating and from a 2D array of particles for the wavelength $\lambda=1 \mathrm{~mm}$ and normal motion of the electron above the target. Here $\gamma=16, M_{x}=3$ (seven periods along the electron trajectory), $M_{y}=5$ (11 periods in each row), $r_{0}=0.1 \mathrm{~mm}$, $\varepsilon=2, h=1 \mathrm{~mm}, d_{x}=d_{y}=2 \mathrm{~mm}$, and $\chi=0$. White dashed lines correspond to the dispersion relation for SPR from a conventional grating.

being that the components of the unit wave vector of the incident plane wave $n_{0 x}, n_{0 y}$ should be substituted by $\beta_{x} / \beta^{2}$, $\beta_{y} / \beta^{2}$. This fact indicates that there is a close analogy between SPR and the optics of diffraction gratings. Similarly to the case of conventional gratings, Eq. (17) can be obtained from the formulas describing the diffraction of plane waves on $2 \mathrm{D}$ gratings [26] directly using Floquet's theorem (the notations have been changed, see Eqs. (6) and (7) in [26]):

$$
\begin{aligned}
& k_{x}=K_{0 x}+2 \pi l_{x} / d_{x}, \\
& k_{y}=K_{0 y}+2 \pi l_{y} / d_{y},
\end{aligned}
$$

where $K_{0 x}$ and $K_{0 y}$ are the components of the wave vector of the incident wave, $k_{x}$ and $k_{y}$ are the components of the wave vector of the diffracted wave, and $l_{x}$ and $l_{y}$ are integers. Keeping in mind that $k_{x, y}=2 \pi n_{x, y} / \lambda, K_{0 x, 0 y}=2 \pi n_{0 x, 0 y} / \lambda$, and replacing $n_{0 x}$ and $n_{0 y}$ by $\beta_{x} / \beta^{2}$ and $\beta_{y} / \beta^{2}$, we immediately obtain Eq. (17), where $l_{x, y}=-s_{x, y}$.

\section{COMPARISON BETWEEN 2D AND CONVENTIONAL GRATINGS}

The angular dependences of spectral-angular distribution of SPR from a conventional grating and from a 2D array of particles are shown in Figs. 5(a) and 5(b) for similar parameters. Figure 5(a) was plotted using the theory for the conventional grating made of infinitely thin and perfectly conducting strips; see [27]. Both targets have the same period in the direction of the electron's motion.

As we see from Fig. 5, the angular distribution from a 2D array is more complex: While the central (at $\phi=0$ ) maxima are at the same angles $\theta$ as those for the conventional grating, they are forked and less intense; also, there are additional maxima at $\phi \neq 0$. The relative intensity of the maxima and the angular width are the same for both gratings.

As we see in Figs. 5(a) and 5(b), the maxima of radiation are in the white dashed lines corresponding to the dispersion relation for conventional SPR. This is because the period is the same and the first equation of the two in Eq. (17) coincides with Eq. (1). The radiation peaks in Fig. 5(a) are concentrated near $\phi=0$, i.e., in the plane $n_{y}=0$ shown in Fig. 1 (this is 
(a)

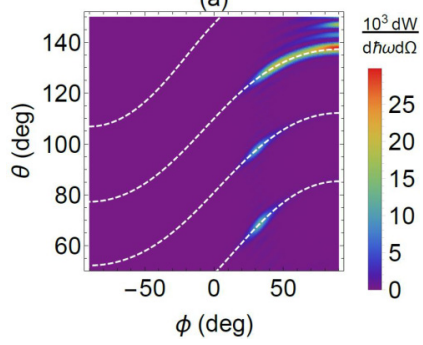

(b)

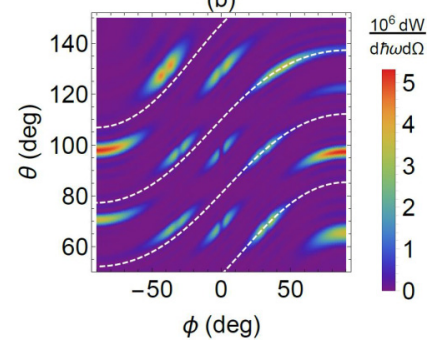

FIG. 6. The same as in Fig. 5, but for oblique movement of electron above the gratings, $\chi=30^{\circ}$.

so due to the decreasing exponent in SPR, which is commonplace for SPR from conventional gratings; see, e.g., [2]).

For 2D array in Fig. 5(b) the peaks, however, are distributed over nonzero azimuthal angles, which is described by the second equation of the two in Eq. (17), $\beta_{y}$ being equal to zero [Fig. 5(b)] or not [Fig. 6(b)].

Note that most peaks for the oblique incidence, Fig. 6(b), are out of the white curves. It corresponds to what we have discussed above in the Introduction: In the case of the 2D array, the dispersion relation for oblique incidence cannot be obtained by a simple amendment of that for a normal incidence, as it takes place for the conventional grating; see Fig. 6(a).

For the conventional grating, the deviation of the electron from motion along the $x$ axis to nonzero $\chi$ leads to the conical effect: All maxima shift to positive $\phi$. For the 2D array, however, all maxima curve and are at both positive and negative $\phi$.

\section{ANGULAR DISTRIBUTION OF RADIATION FOR 2D GRATING}

The changing of spectral-angular distribution of SPR with growth in the value of $\chi$ for the 2D array is shown in Fig. 7. The dashed white curves correspond to the dispersion relations; see Eq. (17). The radiation maxima are at the angles at which these white curves intersect. We see that the obtained dispersion relations define the maxima positions very well.

Some maxima are forked, having the local minimum placed exactly at the intersections of the dispersion relation curves; see, e.g., one at $\phi=0^{\circ}$ and $\theta=90^{\circ}$ in Fig. 7(a).

Also, there is certain inclination of the maxima at $\chi \neq 0$, and the greater $\chi$ is, the more inclined the maxima are. At first sight, the dispersion relations seem not to define the inclinations of the maxima (their fine angular structure), because the intersections of the curves are just the points. Yet, that is not true: These inclinations can also be described by the same dispersion relations. Actually, considering the two expressions in Eq. (17) simultaneously, we find

$$
\lambda\left(s_{x} \beta_{x} d_{y}+s_{y} \beta_{y} d_{x}\right)=d_{x} d_{y}\left(1-n_{x} \beta_{x}-n_{y} \beta_{y}\right) .
$$

Note that Eq. (24) can be obtained immediately by considering the electron-wave synchronism condition in a structure with two periods. That is, any of the systems

$$
\begin{aligned}
& \lambda s_{y}=d_{y}\left(\frac{\beta_{y}}{\beta^{2}}-n_{y}\right), \\
& \lambda\left(s_{x} \beta_{x} d_{y}+s_{y} \beta_{y} d_{x}\right)=d_{x} d_{y}\left(1-n_{x} \beta_{x}-n_{y} \beta_{y}\right),
\end{aligned}
$$
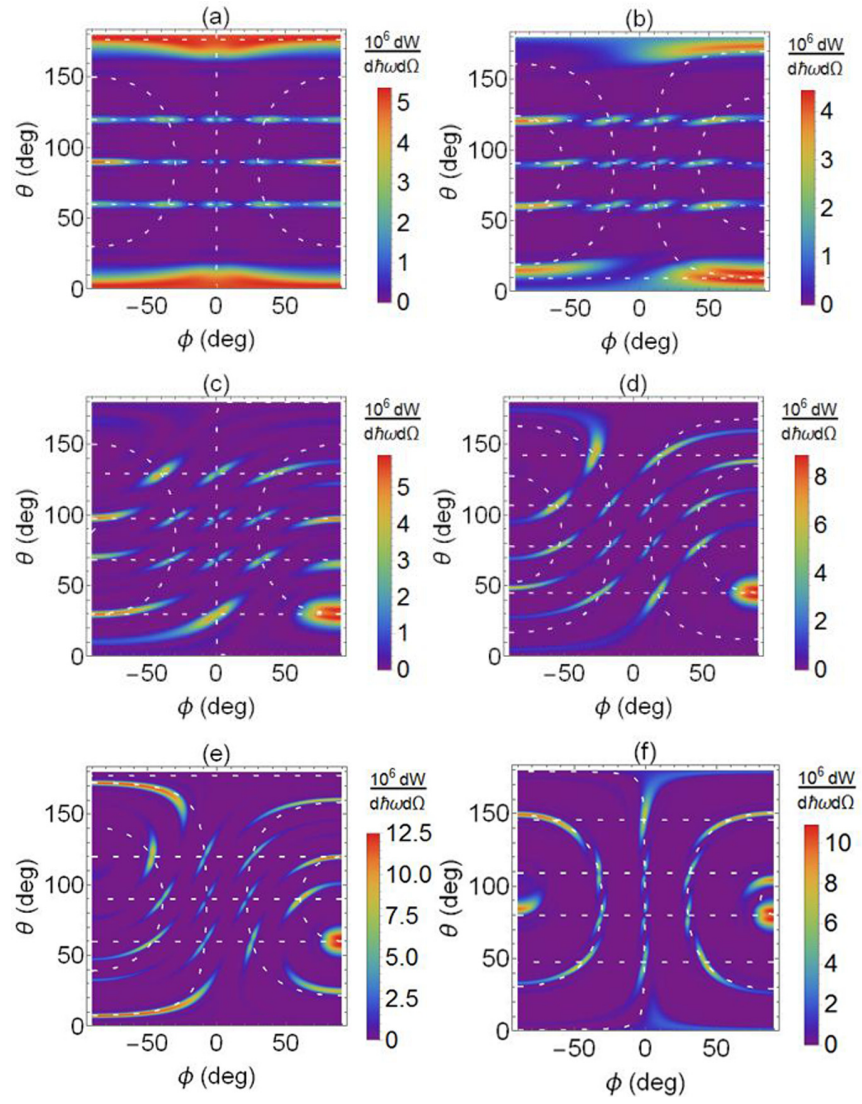

FIG. 7. Spectral-angular distribution of SPR for different $\chi$. All the parameters are the same as in Fig. 5. Here $\chi$ is equal to (a) $0^{\circ}$; (b) $10^{\circ}$; (c) $30^{\circ}$; (d) $45^{\circ}$; (e) $60^{\circ}$; (f) $80^{\circ}$. White dashed curves correspond to dispersion relations given by Eq. (17).

or

$$
\begin{aligned}
& \lambda s_{x}=d_{x}\left(\frac{\beta_{x}}{\beta^{2}}-n_{x}\right), \\
& \lambda\left(s_{x} \beta_{x} d_{y}+s_{y} \beta_{y} d_{x}\right)=d_{x} d_{y}\left(1-n_{x} \beta_{x}-n_{y} \beta_{y}\right),
\end{aligned}
$$

or the initial Eq. (17) as well, is a generalized Smith-Purcell dispersion relation derived for the 2D array considered, which is an arranged array of subwavelength particles, having two periods.

Equation (24) defines the angular spread of radiation near its maxima. In Figs. 8, 7(d), and 7(e) the same angular distributions are shown for $\chi=45^{\circ}, 60^{\circ}$, but while in Figs. 7(d) and 7 (e) the white lines correspond to Eq. (17), in Fig. 8 these white lines are defined by Eq. (24). It is seen that the dispersion relation Eq. (24) perfectly describes the inclination of the SPR maxima: They are extended along the white curves.

In Fig. 8 there are several maxima that do not lie at any of the dashed lines (the most intensive one is the bright spot on the right). That is because the description ("description" rather than "nature"; see below) of this peak differs from the others: It is not because of the periodic structure of the grating (i.e., SPR); it is, better to say, an artifact of diffraction radiation that is emitted directly forward if $\chi=0$, or, if $\chi \neq 0$, either forward or under the direction of mirror reflection.

Interestingly, these peaks are still described by the intersection of the lines from dispersion relations; see Fig. 7. We 
(a)

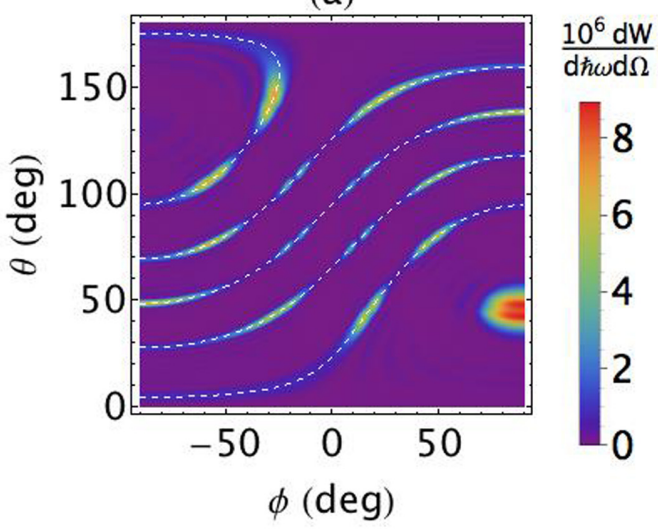

(b)

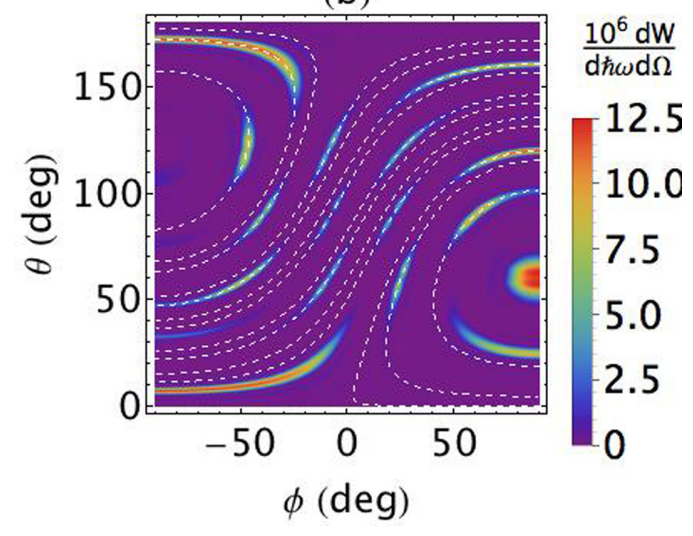

FIG. 8. Spectral-angular distribution of SPR for different $\chi$. All the parameters are the same as in Fig. 5. Here $\chi$ is equal to (a) $45^{\circ}$; (b) $60^{\circ}$. White dashed curves correspond to dispersion relation from Eq. (24).

assume it reflects the deep intrinsic link between diffraction radiation and the Cherenkov radiation. Indeed, Smith-Purcell radiation on the one hand is the special case of diffraction radiation, and on the other hand it formally describes Cherenkov radiation at the "zero" order of diffraction: This is evident from, e.g., Eq. (1) at $s=0$ and $\beta \rightarrow \beta \sqrt{\varepsilon}$ in the presence of the material with $\varepsilon$ being the dielectric permittivity; see also Eq. (5) in [28]. Since, in its turn, diffraction radiation can be understood as a Cherenkov radiation from superluminal sources, see [25], we infer that it is natural when the maxima of diffraction radiation are well described by the generalized Smith-Purcell relation. However, note that this description is very special: Strictly mathematically in Fig. 7 the white lines do not cross at the peaks discussed; i.e., there is no solution of the system of Eq. (17). We see the radiation at these points, however, due to the simple fact that the radiation peaks have the finite width, and when two lines describing positions of maxima approach nearly enough, their tails overlap. At that, the positions of "purely" Smith-Purcell peaks are ideally described by Eq. (17), or the equivalent systems of Eq. (25) or (26).

How does the spatial distribution of radiation change when the number of particles in each row increases? Increasing $M_{y}$ when the period is fixed results in the narrowing of the radiation peaks, until the length of the row along $O Y$ reaches
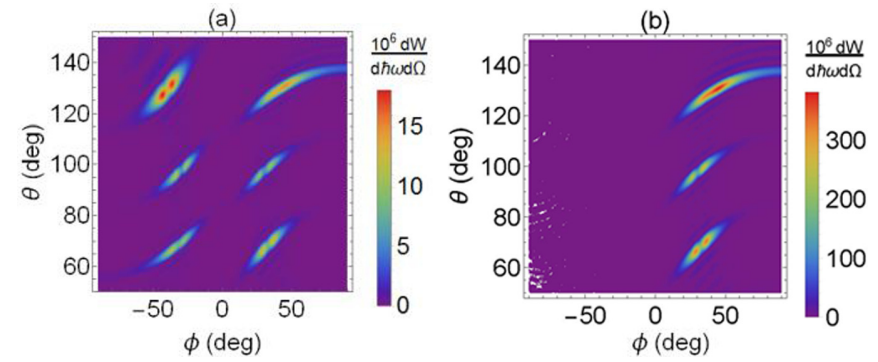

FIG. 9. Angular dependences of spectral-angular distribution of SPR from 2D array for (a) for $M_{y}=10$, i.e., 21 particles in a row $\left(d_{y}=1 \mathrm{~mm}\right)$; (b) for $M_{y}=50$, i.e., 101 particles in a row $\left(d_{y}=\right.$ $0.2 \mathrm{~mm}$ ). Here $\chi=30^{\circ}$ and other parameters are as in Fig. 5 .

the value $\gamma \beta \lambda$-the effective distance to which the Coulomb field of the moving electron extends - and after that ceases to change. If we fix the length of the rows (the transversal size of the 2D array) adding the particles, we turn the rows into continuous strips, and so as a result the diffraction pattern approaches that for a conventional grating; compare Figs. 6(a), 9(a) and 9(b).

\section{CONCLUSION}

To sum up, we have constructed the theory of SmithPurcell radiation from a 2D array-a two-periodic array consisting of subwavelength particles. The theory gives comparatively simple and at the same time fully analytical expressions, describing the distribution of radiation over the angles and frequencies. The size and form of the particles composing the $2 \mathrm{D}$ array are arbitrary, and so are the periods, with two limitations: The particle's size should be subwavelength, and the interaction between separated particles can be neglected. The energy of the electrons is arbitrary and the orientation of the electron's velocity is arbitrary as well, with the restriction that the electron's trajectory is parallel to the surface.

Based on the theory developed, we have derived two dispersion relations [see Eq. (25), or Eq. (26), which is equivalent] that play the role of a generalized Smith-Purcell dispersion relation for the array having two periods. This new dispersion relation describes both spectra and the fine angular structure of the radiation maxima together with their positions: With growth of $\chi$ all maxima shift and incline. Qualitatively, this corresponds to the phenomenon of the diffraction of light on bigratings in optics. As Liang et al. demonstrated recently [29] using numerical simulations, based on the FDTD (finite-difference time-domain) method, for a ribbon electron beam passing above a 2D array (they considered the array composed of holes) the direction of SPR can be steered by adjusting the angle equivalent to the angle $\chi$ in Fig. 3, which perfectly agrees with our results.

Interestingly, unlike the dispersion relation from the arrays/gratings with the only period in one direction at oblique incidence, which is obtained from the conventional SmithPurcell one by simple replace $d \rightarrow d / \cos \chi$, the dispersion relation in the case of $2 \mathrm{D}$ arrays cannot be obtained by a similar rule changing the periods effectively. The whole diffraction pattern proves to be pretty complicated, considerably more complex than that for an array periodic in just one direction. 
The nonrelativistic limit of the theory is applicable for description of electron-photon spectroscopy techniques using electron microscopes; in its relativistic limit the theory forms the basis for the techniques of generating radiation using compact linear accelerators. Using 2D arrays in the capacity of radiating targets should allow the designing of new radiation sources, especially in the $\mathrm{THz}$ range, and developing new diagnostics techniques.

\section{ACKNOWLEDGMENTS}

D.Y.S. and D.I.G. are grateful to the Russian Science Foundation for the financial support of the theoretical part of this research under Grant No. 19-72-00178. A.A.T. is thankful to the Ministry of Education and Science of the Russian Federation for financial support of computer modeling in this research under Project No. FZWG-2020-0032 (2019-1569).
[1] S. J. Purcell and E. M. Smith, Visible light from localized surface charges moving across a grating, Phys. Rev. 92, 1069 (1953).

[2] A. P. Potylitsyn, M. I. Ryazanov, M. N. Strikhanov, and A. A. Tishchenko, Diffraction Radiation from Relativistic Particles, Springer Tracts in Modern Physics Vol. 239 (Springer, Berlin, 2010).

[3] V. P. Shestopalov, The Smith-Purcell Effect (Nova Science Publishers, Commack, NY, 1998).

[4] F. J. García de Abajo, Optical excitations in electron microscopy, Rev. Mod. Phys. 82, 209 (2010).

[5] N. Yamamoto, F. J. García de Abajo, and V. Myroshnychenko, Interference of surface plasmons and Smith-Purcell emission probed by angle-resolved cathodoluminescence spectroscopy, Phys. Rev. B 91, 125144 (2015).

[6] I. Kaminer, S. E. Kooi, R. Shiloh, B. Zhen, Y. Shen, J. J. López, R. Remez, S. A. Skirlo, Y. Yang, J. D. Joannopoulos, A. Arie, and M. Soljačić, Spectrally and Spatially Resolved Smith-Purcell Radiation in Plasmonic Crystals with ShortRange Disorder, Phys. Rev. X 7, 011003 (2017).

[7] Z. Su, F. Cheng, L. Li, and Y. Liu, Complete control of SmithPurcell radiation by graphene metasurfaces, ACS Photonics 6, 1947 (2019).

[8] A. Pizzi, G. Rosolen, L. J. Wong, R. Ischebeck, M. Soljačić, T. Feurer, and I. Kaminer, Graphene metamaterials for intense, tunable, and compact extreme ultraviolet and x-ray sources, Adv. Sci. 7, 1901609 (2020).

[9] K. Tantiwanichapan, X. Wang, A. K. Swan, and R. Paiella, Graphene on nanoscale gratings for the generation of terahertz Smith-Purcell radiation, Appl. Phys. Lett. 105, 241102 (2014).

[10] Z. Wang, K. Yao, M. Chen, H. Chen, and Y. Liu, Manipulating Smith-Purcell Emission with Babinet Metasurfaces, Phys. Rev. Lett. 117, 157401 (2016).

[11] L. Liu, H. Chang, C. Zhang, Y. Song, and X. Hu, Terahertz and infrared Smith-Purcell radiation from Babinet metasurfaces: Loss and efficiency, Phys. Rev. B 96, 165435 (2017).

[12] Y. Song, J. Du, N. Jiang, L. Liu, and X. Hu, Efficient terahertz and infrared Smith-Purcell radiation from metal-slot metasurfaces, Opt. Lett. 43, 3858 (2018).

[13] C. Roques-Carmes, S. E. Kooi, Y. Yang, A. Massuda, P. D. Keathley, A. Zaidi, Y. Yang, J. D. Joannopoulos, K. K. Berggren, I. Kaminer, and M. Soljačić, Towards integrated tunable all-silicon free-electron light sources, Nat. Commun. 10, 3176 (2019).

[14] J. R. M. Saavedra, D. Castells-Graells, and F. Javier Garcia de Abajo, Smith-Purcell radiation emission in aperiodic arrays, Phys. Rev. B 94, 035418 (2016).
[15] N. E. Glass, Enhanced Smith-Purcell radiation from a bigrating surface through multiplasmon excitation, Phys. Rev. A 36, 5235 (1987).

[16] D. Yu. Sergeeva, A. A. Tishchenko, and M. N. Strikhanov, Microscopic theory of Smith-Purcell radiation from 2D photonic crystal, Nucl. Instrum. Methods Phys. Res., Sect. B 402, 206 (2017).

[17] D. Yu. Sergeeva, A. A. Tishchenko, A. S. Aryshev, and M. N. Strikhanov, Smith-Purcell radiation from concave dotted gratings, J. Instrum. 13, C02045 (2018).

[18] D. Yu. Sergeeva, A. A. Tishchenko, and M. N. Strikhanov, Conical diffraction effect in optical and $\mathrm{x}$-ray Smith-Purcell radiation, Phys. Rev. Accel. Beams 18, 052801 (2015).

[19] O. Haeberlé, P. Rullhusen, J.-M. Salomé, and N. Maene, SmithPurcell radiation from electrons moving parallel to a grating at oblique incidence to the rulings, Phys. Rev. E 55, 4675 (1997).

[20] G. A. Naumenko, A. P. Potylitsyn, D. Yu. Sergeeva, A. A. Tishchenko, M. N. Strikhanov, and V. V. Bleko, First experimental observation of conical effect in Smith-Purcell radiation, JETP Lett. 105, 553 (2017).

[21] Yu. Kh. Vekilov and M. A. Chernikov, Quasicrystals, Phys.Usp. 53, 537 (2010).

[22] J. E. S. Socolar and P. J. Steinhardt, Quasicrystals. II. Unit-cell configurations, Phys. Rev. B 34, 617 (1986).

[23] K. V. Lekomtsev, M. N. Strikhanov, and A. A. Tishchenko, Smith-Purcell radiation from a chain of spheres, J. Phys.: Conf. Ser. 236, 012023 (2010).

[24] A. A. Tishchenko and D. Yu. Sergeeva, Near-field resonances in photon emission via interaction of electrons with coupled nanoparticles, Phys. Rev. B 100, 235421 (2019).

[25] A. P. Potylitsyn, D. Yu. Sergeeva, M. N. Strikhanov, and A. A. Tishchenko, Diffraction radiation from a charge as radiation from a superluminal source in a vacuum, Phys.-Usp. 63, 303 (2020).

[26] R. Bräuer and O. Bryngdahl, Electromagnetic diffraction analysis of two-dimensional gratings, Opt. Commun. 100, 1 (1993).

[27] A. P. Potylitsyn, Transition radiation and diffraction radiation. Similarities and differences, Nucl. Instrum. Methods Phys. Res., Sect. B 145, 169 (1998).

[28] A. A. Ponomarenko, K. V. Lekomtsev, A. A. Tishchenko, M. N. Strikhanov, and J. Urakawa, CST simulation of THz radiation from a channel with periodically variable radius, Nucl. Instrum. Methods Phys. Res., Sect. B 355, 160 (2015).

[29] L. Liang, W. Liu, Y. Liu, Q. Jia, L. Wang, and Y. Lu, Multi-color and multidirectional-steerable Smith-Purcell radiation from 2D sub-wavelength hole arrays, Appl. Phys. Lett. 113, 013501 (2018). 